DEGRADAÇÃO DO ANTIBIÓTICO METRONIDAZOL POR PROCESSOS OXIDATIVOS AVANÇADOS

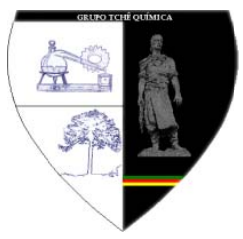

\title{
DEGRADATION OF THE ANTIBIOTIC METRONIDAZOLE BY ADVANCED OXIDATIVE PROCESSES
}

\author{
SILVA, Schirlei Diana Kleinubing ${ }^{1}$; HASAN, Salah Din Mahmud ${ }^{2}$; ROSA, Mauricio Ferreira ${ }^{1, *}$ \\ ${ }^{1}$ Universidade Estadual do Oeste do Paraná, Programa de Pós-Graduação em Ciências Ambientais, Rua da \\ Faculdade 645, CEP85903-000, Toledo - PR, Brasil \\ (fone: +55 4533797074 ) \\ ${ }^{2}$ Universidade Estadual do Oeste do Paraná, Programa de Pós-Graduação em Engenharia Química, Rua \\ da Faculdade 645, CEP 85903-000, Toledo - PR, Brasil \\ (fone: +55 4533797000 ) \\ * Autor correspondente \\ e-mail: mauricio.rosa@unioeste.br
}

Received 14 February 2017; received in revised form 01 March 2017; accepted 24 March 2017

\section{RESUMO}

A indústria farmacêutica é um dos setores econômicos que mais crescem no mundo. A existência de medicamentos para as mais diferentes enfermidades fez o uso destes produtos crescer vertiginosamente nos últimos anos. Quando ingeridos estes compostos são parcialmente absorvidos pelo organismo, e parte excretada na sua forma original. As estações de tratamento de esgoto (ETE), com processos baseados na degradação biológica, não conseguem remover estes fármacos da água. Ao ser devolvida para o ambiente, ou destinada ao consumo humano, esta água contém pequenas quantidades destes compostos, que podem causar efeitos deletérios, tanto para o ser humano quanto para o ambiente. Nesta situação o emprego de métodos fotocatalíticos podem ser uma importante alternativa na remoção destes contaminantes do meio aquático. Este trabalho estudou a fotodegradação do antibiótico metronidazol (MNZ) em solução aquosa por métodos fotolíticos e fotocatalíticos (POA) empregando luz ultravioleta, peróxido de hidrogênio $\left(\mathrm{H}_{2} \mathrm{O}_{2}\right)$ e dióxido de titânio $\left(\mathrm{TiO}_{2}\right)$. Os ensaios de fotocatálise na presença de $\mathrm{H}_{2} \mathrm{O}_{2}$ foram realizados nos valores de $\mathrm{pH}$ 4, 7 e 10. Nos experimentos de fotocatálise heterogênea foi realizado um planejamento experimental $2^{3}$ para avaliar a influência das variáveis $\mathrm{pH}$, concentração de $\mathrm{H}_{2} \mathrm{O}_{2}$ e concentração do catalisador na fotodegradação do fármaco, que foi monitorada por espectrofotometria na região do UV-VIS. O modelo cinético de pseudo-primeira ordem descreveu apropriadamente o comportamento da fotodegração do fármaco. Nos resultados de fotocatálise unicamente na presença de $\mathrm{H}_{2} \mathrm{O}_{2}$, a maior taxa de degradação obtida foi de $8,66 \%$ em pH 4. Nos ensaios de fotocatálise heterogênea a maior taxa de degradação do MNZ foi de $72,1 \%$ quando empregou-se $\mathrm{TiO}_{2}$ na concentração de $100 \mathrm{mg} \cdot \mathrm{L}^{-1}, \mathrm{H}_{2} \mathrm{O}_{2}$ na concentração de 1000 $\mathrm{mg} \cdot \mathrm{L}^{-1} \mathrm{em} \mathrm{pH}$ 10. As análises estatísticas dos resultados mostraram que nos experimentos de fotocatálise heterogênea com $\mathrm{TiO}_{2}$ apenas a interação $\mathrm{pH} / \mathrm{H}_{2} \mathrm{O}_{2}$ não teve efeito significativo. Ensaios de citotoxicidade empregando Artemia salina verificou-se uma redução de $90 \%$ na toxicidade da amostra após o tratamento fotocatalítico.

Palavras-chave: Contaminantes emergentes; fármacos;remediação

\section{ABSTRACT}

The pharmaceutical industry is one of the fastest growing economic sectors in the world. The existence of medicines for the most different diseases has made the use of these products grow vertiginously in recent years. When ingested these compounds are partially absorbed by the body, and part excreted in its original form. Sewage treatment plants (ETS), with processes based on biological degradation, can not remove these drugs from water. When it is returned to the environment, or intended for human consumption, this water contains small amounts of these compounds, which can cause deleterious effects to both humans and the environment. In this situation the use of photocatalytic methods can be an

PERIÓDICO TCHÊ QUÍMICA • www.periodico.tchequimica.com • Vol. 14 N. 28

- ISSN 1806-0374 (impresso) • ISSN 1806-9827 (CD-ROM) • ISSN 2179-0302 (meio eletrônico)

(C) 2017. Porto Alegre, RS. Brasil 
important alternative in the removal of these contaminants from the aquatic environment. This work studied the photodegradation of the antibiotic metronidazole (MNZ) in aqueous solution by photolytic and photocatalytic methods (POA) using ultraviolet light, hydrogen peroxide $\left(\mathrm{H}_{2} \mathrm{O}_{2}\right)$ and titanium dioxide $\left(\mathrm{TiO}_{2}\right)$. To investigate the photocatalytical process, an experimental design $2^{3}$ was performed to evaluate the influence of the variables $\mathrm{pH}$, hydrogen peroxide concentration and $\mathrm{TiO}_{2}$ concentration over the photodegradation of drug, which was spectrophotometrycally monitored in the UV-VIS region. The kinetic model of pseudofirst order described quite well the degradation behavior of the drug. The photocatalytical process only in presence of $\mathrm{H}_{2} \mathrm{O}_{2}$, the highest rate of degradation obtained was $8.66 \%$ at $\mathrm{pH} 4$. In the heterogeneous photocatalysis experiments the highest degradation rate of the $\mathrm{MNZ}$ was $72.1 \%$ when $\mathrm{TiO}_{2}$ was used in concentration of $100 \mathrm{mg} \cdot \mathrm{L}^{-1}, \mathrm{H}_{2} \mathrm{O}_{2}$ at the concentration of $1000 \mathrm{mg} \cdot \mathrm{L}^{-1}$ at $\mathrm{pH} 10$. The statistical analysis of the results showed that in the heterogeneous photocatalysis experiments with $\mathrm{TiO} 2$ only the $\mathrm{pH} / \mathrm{H}_{2} \mathrm{O}_{2}$ interaction had no significant effect. Cytotoxicity assays employing Artemia salina as bioindicator showed a $90 \%$ reduction in sample toxicity after the photocatalytical treatment.

Keywords: emergent contaminants; pharmaceuticals; remediation

\section{INTRODUÇÃO}

Grande quantidade de medicamentos é produzida anualmente e utilizados na medicina humana e veterinária, representando um fator de grande preocupação em contaminação ambiental. Um ponto importante acerca da exposição ambiental por resíduos de medicamentos, é que estes são desenvolvidos para que tenham ótima estabilidade ou meia-vida prolongada. Este fato, aliado às propriedades físico-químicas, conferem a estes compostos químicos elevada tendência a bioacumulação. Segundo Sorensen e colaboradores (2009), 30\% dos fármacos são lipofílicos, sedimentando-se em ambientes aquáticos ou transferindo-se para a fase biótica.

Estudos revelam que as classes de medicamentos mais impactantes são os antibióticos, os hormônios e os antidepressivos. Entre os antibióticos, $76,6 \%$ apresentam inerente risco ambiental (Binieka et al., 2005).

Os antibióticos podem causar mudanças irreversíveis, a longo prazo, no genoma de microrganismos, tornando-os resistentes a estas substâncias, mesmo em baixas concentrações. Efeitos adversos no sistema reprodutivo de seres humanos e animais, além de efeitos carcinogênicos e mutagênicos, também têm sido relatados (Díaz-Cruz et al., 2003; Boxall, 2004; Reis Filho et al., 2006; Bila \& Dezotti, 2003).

Os antibióticos utilizados na medicina humana são introduzidos no ambiente através da excreção pela urina e fezes e levados ao sistema de esgoto, chegando às Estações de Tratamento de Esgoto (ETEs), onde são submetidos aos processos convencionais de tratamento.
Contudo, esses processos, geralmente baseados na degradação biológica, não são eficientes para completa remoção de fármacos residuais, em decorrência de suas complexas estruturas químicas nem sempre passíveis de biodegradação, levando à contaminação de águas superficiais (Melo et al., 2009).

A utilização de esterco animal contaminado com resíduos de medicamentos, para fertilização de solos agrícolas, representa uma importante via de contaminação ambiental, visto que há possibilidade de lixiviação e contaminação de águas profundas e do próprio solo (HallingSorensen et al., 1998).

Resíduos de antibióticos têm sido detectados em níveis elevados em efluentes hospitalares $\left(0,003-101 \mu \mathrm{g} \mathrm{L}^{-1}\right)$ e efluentes de indústrias farmacêuticas $\left(0,026-43900 \mu \mathrm{g} \mathrm{L}^{-1}\right)$. Os efluentes de ETEs normalmente também contém elevadas concentrações de antibióticos $\left(0,002-64 \mu \mathrm{g} \mathrm{L}^{-1}\right)$, comprovando a ineficiência dos processos de tratamento normalmente utilizados (Kummerer, 2009).

Entre os antibióticos, merece destaque o composto metronidazol, detectado em águas residuais de efluentes hospitalares na faixa de 0,1 a $90,2 \mu \mathrm{g} \mathrm{L}^{-1}$ (Lindeberg et al., 2004), em águas superficiais (agua de rio) na faixa de 2 a $24 \mathrm{ng} \mathrm{L}^{-1}$ (Kasprzyk-Hordern et al., 2008) e em águas subterrâneas, indicando que ele não é removido pelos processos convencionais de tratamento empregados nas ETEs, podendo comprometer o equilíbrio ambiental, bem como elevar o risco de desenvolvimento de bactérias resistentes a este composto no ambiente. 
O metronidazol (MNZ), de nome químico 1( $\beta$-hidroxietil)-2-metil-5-nitroimidazol (Figura 1), pertence à classe dos nitroimidazóis, extensamente usado no tratamento de infecções causadas por bactérias anaeróbicas e protozoários, como a Giardia lamblia e Trichomonas vaginalis.<smiles>Cc1ncc([N+](=O)[O-])n1CCO</smiles>

Figura 1 - Fórmula estrutural do metronidazol (MNZ)

Este composto apresenta baixa biodegradabilidade e solubilidade em água (Shemer et al., 2006) favorecendo seu acúmulo nos ambientes aquáticos. Estudos relatam efeitos mutagênicos deste antibiótico em bactérias, nas quais provocam alterações ao nível do DNA(Çavas \& Ergene-Gözükara, 2005), podendo exercer efeitos genotóxicos e carcinogênicos em outros organismos, tornando fundamental sua completa remoção dos efluentes aquáticos.

\section{Os Processos Oxidativos Avançados} (POAs) têm sido uma alternativa ou complemento aos processos convencionais de tratamento de efluentes, uma vez que os radicais hidroxila gerados possuem elevada reatividade $\mathrm{e}$ uma menor seletividade para remoção de poluentes recalcitrantes/refratários, mostrando-se bastante eficazes no processo de descontaminação ambiental (Dantas et al., 2010). Estes radicais são produzidos a partir de agentes oxidantes como 0 peróxido de hidrogênio $\left(\mathrm{H}_{2} \mathrm{O}_{2}\right)$, combinados ou não com catalisadores metálicos ou semicondutores e irradiação UV. Com a utilização destes processos, espera-se que os compostos orgânicos sejam oxidados a espécies intermediárias menos tóxicas e mais biodegradáveis, ou até mineralizados a $\mathrm{CO}_{2}, \mathrm{H}_{2} \mathrm{O}$ e sais inócuos (Dantas et al., 2010).

Esse radical possui a capacidade de reagir com água e íons hidroxila para formação de radical hidroxila, peróxido de hidrogênio e outras espécies reativas. $\mathrm{O}$ radical ${ }^{\circ} \mathrm{OH}$ e as outras substâncias formadas apresentam alto poder oxidante, e em contato com moléculas orgânicas podem levar à sua oxidação e, idealmente, sua mineralização (Xavier etal., 2005; Fujishima etal., 2008).

$\mathrm{O}$ mecanismo de funcionamento do $\mathrm{TiO}_{2}$ como fotocatalisador baseia-se na geração do par elétron-lacuna positiva, através da absorção de energia.Sob irradiação, elétrons da banda de valência recebem energia suficiente para transpor a lacuna de energia do $\mathrm{TiO}_{2}$ e podem, desta forma, ser transferidos para a banda de condução. As lacunas positivas são fortes agentes oxidantes e são responsáveis pela mineralização de vários compostos orgânicos (Silva et al., 2010).

$\mathrm{O} \mathrm{TiO}_{2}$ é amplamente utilizado devido ao custo relativamente baixo, à baixa solubilidade em água, à fotoestabilidade, à estabilidade química em ampla faixa de $\mathrm{pH}$, à não toxicidade, à possibilidade de inativação sobre superfícies sólidas e a ativação pela luz solar, o que diminui consideravelmente os custos do processo (Nogueira et al., 1997).

Em relação ao MNZ, estudos utilizando o processo de fotólise direta e de fotólise com a utilização do $\mathrm{H}_{2} \mathrm{O}_{2}$ foram realizados. Shemer e colaboradores (2006) estudaram a fotodegradação do MNZ e apenas $6 \%$ do antibiótico foi degradado. No entanto, quando combinaram $\mathrm{O}$ processo com $\mathrm{H}_{2} \mathrm{O}_{2}$, obtiveram taxas de degradação superiores a $67 \%$.

A grande preocupação em relação aos efeitos dos antibióticos nos ecossistemas aquáticos tem despertado interesse por estudos dos impactos causados nesses ambientes. Análises ecotoxicológicas vêm sendo empregadas no monitoramento de efluentes, para avaliar a qualidade dos mesmos e minimizar o impacto ambiental, além de verificar a eficiência dos processos de tratamento. Os ensaios ecotoxicológicos investigam os efeitos de poluentes químicos em organismos aquáticos e, consequentemente, no ambiente como um todo.

A Artemia sp. é um microcrustáceo empregado para monitoramento da remoção da carga tóxica de efluentes aquáticos. Ela pertence ao filo Arthropoda, classe Crustácea, subclasse Branquiopoda, ordem Anostraca, família Artemidae e Gênero Artemia (Leach, 1819). Possui distribuição cosmopolita e caráter extremamente eurialino (Veiga \& Vital, 2002). Além disso, os organismos desse gênero atuam como elo trófico entre as comunidades planctônicas e as cadeias superiores. 
Por se tratar de um microrganismo de fácil manutenção em condições de laboratório e de ampla distribuição, a Artemia sp. tem sido largamente utilizada em testes de toxicidade. Estes testes consistem na exposição dos náuplios na fase II ou III durante 24 e/ou 48 horas a concentrações crescentes da amostra que se pretende testar com análise do número de organismos mortos ao final do período de exposição (Veiga \& Vital, 2002; CETESB, 1987).

Este trabalho tem como objetivo estudar as metodologias fotolíticas e fotocatalíticas para remoção do antibiótico MNZ do ambiente, diminuindo o impacto causado nos ecossistemas aquáticos e o risco potencial de contaminação da água de abastecimento público.

\section{PARTE EXPERIMENTAL}

\section{Reagentes}

O metronidazol (MNZ) foi adquirido junto a empresa Sigma-Aldrich com grau de pureza $99,9 \%$. Para os ensaios de oxidação foi utilizada uma solução de $\mathrm{H}_{2} \mathrm{O}_{2}$ (Anidrol) padronizado utilizando a técnica de titulação permanganométrica (Vogel, 1992). Para os ajustes de $\mathrm{pH}$ utilizou-se hidróxido de sódio $(\mathrm{NaOH})$ (FMAIA) e ácido clorídrico $(\mathrm{HCl})$ (Labsynth) ambos grau P.A.. Os ensaios de fotocatálise foram realizados utilizando-se $\mathrm{TiO}_{2}$ obtido junto a Sigma-Aldrich (pureza de 99,9\%).

\section{Métodos analíticos}

Foi utilizado um espectrofotômetroShimadzu UV-1601PC para a obtenção dos espectros na região UV-Vis das amostras iniciais de MNZ (10 mg $\left.\cdot \mathrm{L}^{-1}\right)$, bem como das amostras submetidas aos processos de degradação. Os espectros de absorção foram obtidos em intervalos de 10 minutos na faixa de comprimento de onda entre 200 e $400 \mathrm{~nm}$.

\section{Reator fotoquímico}

Foi utilizado um foto-reator lab-made (SCHNEIDER et al., 2014) equipado com uma lâmpada de vapor de mercúrio $(\mathrm{Hg})$ de alta pressão de $125 \mathrm{~W}$ de potência, cujo bulbo externo foi removido para evitar o efeito de filtro da radiação ultravioleta (UV). A lâmpada de vapor de $\mathrm{Hg}$ foi instalada a uma distância de 11 $\mathrm{cm}$ da amostra.

\section{Preparo da amostra}

A solução estoque de MNZ foi preparada dissolvendo-se $0,1 \mathrm{~g}$ do antibiótico em $100 \mathrm{~mL}$ de água destilada, obtendo-se uma solução estoque de concentração $1,0 \mathrm{~g} \mathrm{~L}^{-1}$. A solução foi submetida a um banho ultrassônico durante 25 minutos, a fim de garantir a completa solubilização da amostra. As soluções de trabalho, na concentração de $10 \mathrm{mg} \cdot \mathrm{L}^{-1}$, foram preparadas a partir da diluição da solução estoque.

Os ensaios foram realizados em $250 \mathrm{~mL}$ de solução aquosa contendo $10 \mathrm{mg} \cdot \mathrm{L}^{-1}$ de $\mathrm{MNZ}$, as quais foram irradiadas durante 60 minutos em foto-reator. Em intervalos de 10 minutos eram retiradas alíquotas de 3,0 mL para realização de leituras espectrais utilizadas para o acompanhamento da cinética de reação.

Foram feitas medidas de absorbância das amostras no tempo inicial $\left(A_{0}\right)$ e final $\left(A_{f}\right)$ e relacionadas para a construção dos gráficos de $\ln \left(A_{0} / A_{f}\right)$ vs tempo (minutos) empregando-se os valores de absorção localizado no comprimento de onda de $320 \mathrm{~nm}$. Os gráficos foram traçados empregando-se o programa Microsoft Excel ${ }^{\circledR}$ e o tratamento estatístico foi realizado por meio do software estatístico Statistica ${ }^{\circledR}$.

\subsection{Fotólise utilizando luz UV, influência do $p H$}

Foram realizados os ensaios de fotodegradação empregando-se luz UV em $\mathrm{pH} 4$, 7 e 10 , ajustados com soluções $0,01 \mathrm{~mol} \mathrm{~L}^{-1}$ de $\mathrm{HCl}$ e $0,01 \mathrm{~mol} \mathrm{~L}^{-1}$ de $\mathrm{NaOH}$.

\subsection{Fotólise utilizando $\mathrm{H}_{2} \mathrm{O}_{2}$ e luz UV, influência do $\mathrm{pH}$}

Os ensaios de fotodegradação utilizando o $\mathrm{H}_{2} \mathrm{O}_{2}$ foram realizados em $\mathrm{pH} 4,7$ e 10 e avaliadas as concentrações de $600 \mathrm{mg} \cdot \mathrm{L}^{-1} \mathrm{e}$ $1000 \mathrm{mg} \cdot \mathrm{L}^{-1}$ de $\mathrm{H}_{2} \mathrm{O}_{2}$, com base em estudos preliminares.

4.3. Fotocatálise utilizando $\mathrm{TiO}_{2}$ e luz UV, ausência ou presença de $\mathrm{H}_{2} \mathrm{O}_{2}$ e influência do $\mathrm{pH}$

Para os estudos de fotocatálise utilizando o catalisador $\mathrm{TiO}_{2}$, avaliaram-se os perfis de degradação nos pH's 4, 7 e 10 e a presença de 
$\mathrm{H}_{2} \mathrm{O}_{2}$. Foram preparadas soluções aquosas de MNZ (10 $\left.\mathrm{mg} \cdot \mathrm{L}^{-1}\right)$ contendo $\mathrm{TiO}_{2}$ nas concentrações de 50 e $100 \mathrm{mg} \cdot \mathrm{L}^{-1}$. O pH foi ajustado para os valores desejados e a mistura foi mantida ao abrigo da luz, com agitação constante, durante 30 minutos até que o equilíbrio de adsorção fosse atingido. Adicionouse a quantidade necessária de $\mathrm{H}_{2} \mathrm{O}_{2} \mathrm{e}$, em seguida, a amostra foi submetida a irradiação UV, coletando-se alíquotas da solução irradiada em intervalos de 10 minutos. Para realização das leituras espectrais a amostra foi filtrada em filtros-seringa com poros de $0,22 \mu \mathrm{m}$ (Chromafil PVDF-20/15 MS), sendo transferida em seguida para um cubeta de quartzo de $1,0 \mathrm{~cm}$ de caminho óptico.

\subsection{Planejamento experimental $2^{3}$}

Para os experimentos de fotocatálise utilizando $\mathrm{TiO}_{2}$, foi realizado um planejamento experimental $2^{3}$, com triplicata no ponto central, a fim de avaliar os efeitos das variáveis $\mathrm{pH}$, concentração de $\mathrm{H}_{2} \mathrm{O}_{2}$ e concentração do catalisadorna degradação do MNZ. Os níveis das variáveis do processo estão representados na Tabela 1.

Os 11 experimentos do planejamento foram realizados de maneira aleatória. As três variáveis de processo variaram entre si nos dois níveis $(-1$ e +1) de concentração dos reagentes e do $\mathrm{pH}$ inicial, sendo realizada uma triplicata no ponto central, com objetivo de calcular o erro experimental e aumentar o grau de liberdade do modelo, conforme apresentado pela Tabela 3.

A variável resposta em todos os experimentos foi o percentual de redução da banda (\%Red) centrada em $320 \mathrm{~nm}$, calculado utilizando a Equação 1.

$$
\% \operatorname{Red}=\left[1-\left(A_{f} / A_{0}\right)\right] \times 100
$$

Os resultados dos experimentos obtidos no planejamento experimental foram avaliados por meio do software estatístico Statistica ${ }^{\circledR}$, para dois níveis de variação, sendo realizadas análises dos efeitos, interações, superfície de resposta e análise de variância (ANOVA).

\section{Avaliação da toxicidade utilizando Artemia salina}

A metodologia utilizada para os ensaios de toxicidade utilizando Artemia salina foi baseada em Meyer e colaboradores (1982), em Nascimento e colaboradores (1999) e nas normas da CETESB-SP (1991).

Dez náuplios de $A$. salina que apresentavam motilidade após transferidos para tubos de ensaio foram expostos por 48 horas a soluções aquosas de MNZ na concentração de $10 \mathrm{mg} \cdot \mathrm{L}^{-1}$ sem tratamento (controle positivo), outra de MNZ obtida após o tratamento que apresentou as melhores taxas de degradação do antibiótico (amostra) e água destilada (controle negativo). Os testes foram feitos em duplicata. Após 48 horas de exposição, foi feita a contagem de náuplios vivos e mortos, sendo considerados vivos todos aqueles que apresentassem qualquer tipo de movimento quando observados próximos a uma fonte luminosa.

\section{RESULTADOS E DISCUSSÃO:}

\section{Fotólise utilizando luz UV, influência do pH}

Para avaliar a influência do $\mathrm{pH}$ no processo de degradação do antibiótico MNZ, foram preparadas soluções aquosas do fármaco, na concentração de $10 \mathrm{mg} \cdot \mathrm{L}^{-1}$, com $\mathrm{pH}$ ajustado em 4,7 e 10. Após 60 minutos de irradiação foi calculado, conforme a Equação 10, o percentual de redução da intensidade da banda centrada em $320 \mathrm{~nm}$.

No processo de fotodegradação, acompanharam-se os espectros de absorção em intervalos de 10 minutos. Todos os ensaios apresentam comportamento espectrofotométrico semelhante, diferindo-se apenas pelos valores de percentual de redução da absorbância. Foram obtidos, ao final de 60 minutos de irradiação, os valores de redução de $8,66 \%, 3,87 \%$ e $1,77 \%$ para valores de $\mathrm{pH} 4,7$ e 10, respectivamente.

Para obter a cinética da reação os dados experimentais de degradação do MNZ foram ajustados usando o modelo de pseudoprimeira ordem, representado pela Equação 2.

$$
\ln \left(\mathrm{A}_{0} / \mathrm{A}_{\mathrm{f}}\right)=k \mathrm{t}
$$

$\mathrm{Na}$ Figura 2 estão representados os gráficos de $\ln \left(A_{0} / A\right)$ em função do tempo para os três experimentos.

É possível verificar que o ensaio em $\mathrm{pH} 4$ é o que apresenta maior valor de $k$, da ordem de 
$1,5 \times 10^{-3} \mathrm{~min}^{-1}$, o que significa que o MNZ é degradado mais rapidamente neste $\mathrm{pH}$ do que nos demais. Observando os valores dos coeficientes de correlação $\left(R^{2}\right)$, representados no Tabela 3 , é possível afirmar que as relações lineares entre $\ln \left(A_{0} / A\right)$ versus o tempo seguem uma cinética de pseudoprimeira ordem até 60 minutos de reação.

Shemer e colaboradores (2006), obtiveram uma porcentagem de remoção de $12 \%$ em pH 6,0 . Esta diferença pode estar relacionada ao tipo de reator empregado, onde a superfície e a profundidade da solução a ser degradada eram muito menores do que as utilizadas no desenvolvimento desse trabalho, o que permite uma maior incidência de luz UV e, consequentemente, um maior percentual de degradação do fármaco.

Em relação a influência do $\mathrm{pH}$ na fotodegradação utilizando luz UV, Prados-Joya e colaboradores (2011), avaliaram a influência do $\mathrm{pH}$ no processo de fotodegradação de compostos nitroimidazólicos. Segundo estes autores, o pH da solução provoca variações no coeficiente de absorção molar e na constante de velocidade da reação. No entanto, estas mudanças dependem do $\mathrm{pK}_{\mathrm{a}}$ do fármaco. Para o MNZ, os autores verificaram um aumento na fotodegradação em $\mathrm{pH}$ menor que 4 , o mesmo comportamento foi observado neste trabalho.

\section{Fotólise utilizando $\mathrm{H}_{2} \mathrm{O}_{2}$ e luz UV, influência do $\mathrm{pH}$}

A influência da adição de $\mathrm{H}_{2} \mathrm{O}_{2}$ no processo de fotodegradação foi avaliada em soluções aquosas de MNZ com pH's previamente ajustados em 4, 7 e 10.

A Tabela 3 apresenta os valores de \%Red nas diferentes condições experimentais estudadas. Foram avaliadas as concentrações de 600 e $1000 \mathrm{mg} \cdot \mathrm{L}^{-1}$ de $\mathrm{H}_{2} \mathrm{O}_{2}$, em pH 4, 7 e 10, bem como os valores obtidos nos experimentos de fotodegradação sem adição de $\mathrm{H}_{2} \mathrm{O}_{2}$.

Para a determinação das constantes cinéticas $(k)$ das reações, foram traçados os gráficos de $\ln \left(A_{0} / A\right)$ em função do tempo para todos os experimentos realizados, acompanhando-se o comportamento da banda centrada em $320 \mathrm{~nm}$, o que permitiu a obtenção dos valores de $\mathrm{R}^{2}$.
Comparando-se os processos de fotodegradação em presença ou ausência de $\mathrm{H}_{2} \mathrm{O}_{2}$, verifica-se que no $\mathrm{pH} 10$ a cinética de degradação do processo utilizando o catalisador $\left(k=1,23 \times 10^{-2} \mathrm{~min}^{-1}\right)$ é muito superior ao processo de fotodegradação na ausência do catalisador $\left(k=2,97 \times 10^{-4} \mathrm{~min}^{-1}\right)$. Shemer $\mathrm{e}$ colaboradores (2006), também verificaram um aumento na degradação do $\mathrm{MNZ}$ quando $\circ \mathrm{H}_{2} \mathrm{O}_{2}$ foi adicionado ao processo. Isto ocorre em virtude do elevado rendimento quântico do $\mathrm{H}_{2} \mathrm{O}_{2}$ para se decompor e gerar radicais hidroxila $\left(\mathrm{HO}^{\circ}\right)$ altamente reativos, quando expostos a radiação UV (Sánchez-Polo et al., 2012).

A Figura 3 apresenta os efeitos das variáveis $\mathrm{pH}$ e $\mathrm{H}_{2} \mathrm{O}_{2}$ na análise estatística do planejamento experimental $2^{3}$. Observa-se um maior percentual de redução da absorbância em $\mathrm{pH} 10$ e na maior concentração de $\mathrm{H}_{2} \mathrm{O}_{2}$ estudada (1000 $\left.\mathrm{mg} \cdot \mathrm{L}^{-1}\right)$. Segundo Melo e colaboradores (2009), em meio alcalino a dissociação do $\mathrm{H}_{2} \mathrm{O}_{2}$ é favorecida. SegundoAndreozzi, Raffaele \& Nicklas (2003) e Cisneros, Espinoza e Litter (2002), isso se deve à formação do ânion $\mathrm{HO}_{2}^{-}$, que apresenta maior coeficiente de absortividade molar do que o próprio peróxido de hidrogênio.

\section{Fotocatálise utilizando $\mathrm{TiO}_{2}$ e luz UV}

\subsection{Efeito da concentração de $\mathrm{TiO}_{2}$}

$\mathrm{O}$ efeito da concentração de $\mathrm{TiO}_{2}$ sobre a degradação do MNZ na concentração de 10 $\mathrm{mg} \cdot \mathrm{L}^{-1}$, foi avaliada em $\mathrm{pH} 10$. Na Figura 4estão representados os gráficos de $\ln \left(A_{0} / A_{f}\right)$ em função do tempo para as concentrações de $50 \mathrm{mg} \cdot \mathrm{L}^{-1} \mathrm{e}$ $100 \mathrm{mg} \cdot \mathrm{L}^{-1} \mathrm{de} \mathrm{TiO}_{2}$.

É possível verificar na Tabela 4 que o $\mathrm{TiO}_{2}$ na concentração de $100 \mathrm{mg} \cdot \mathrm{L}^{-1}$ apresenta um maior valor de $k\left(1,62 \times 10^{-2} \mathrm{~min}^{-1}\right)$, o que significa que o MNZ é degradado mais rapidamente nesta concentração de catalisador. Observando os valores de \%Red para as concentrações avaliadas, 50 e $100 \mathrm{mg} \cdot \mathrm{L}^{-1}$, verifica-se uma maior eficiência de degradação, $62,23 \%$, após 60 minutos de exposição à luz UV, quando a concentração de $100 \mathrm{mg} \cdot \mathrm{L}^{-1}$ foi utilizada.

Okhovate colaboradores (2015), analisaram o efeito da concentração de 500 $\mathrm{mg} \cdot \mathrm{L}^{-1}, 1500 \mathrm{mg} \cdot \mathrm{L}-1$ e $3000 \mathrm{mg} \cdot \mathrm{L}^{-1}$ do catalisador e obtiveram uma melhor taxa de degradação, em torno de $80 \%$, após 60 minutos de exposição à 
luz UV, para a menor concentração de catalisador avaliada.

Farzadkia e colaboradores (2015), também avaliaram a eficiência de degradação do $\mathrm{TiO}_{2}$ nas concentrações $500 \mathrm{mg} \cdot \mathrm{L}^{-1}, 1500 \mathrm{mg} \cdot \mathrm{L}^{-1} \mathrm{e}$ $3000 \mathrm{mg} \cdot \mathrm{L}^{-1} \mathrm{e}$ obtiveram resultados semelhantes, ou seja, observaram uma melhor eficiência na degradação quando a concentração $500 \mathrm{mg} \cdot \mathrm{L}^{1}$ foi empregada.

Segundo estes autores, o aumento na concentração do $\mathrm{TiO}_{2}$ reduz a porcentagem de degradação, devido ao aumento da turbidez da solução e, consequentemente, a redução do poder de penetração da luz UV.

\subsection{Efeito do $\mathrm{pH}$}

Para avaliar o efeito do $\mathrm{pH}$ na degradação fotocatalítica do MNZ, utilizou-se uma concentração fixa do catalisador, $100 \mathrm{mg} \cdot \mathrm{L}^{-1}$, variando-se $\mathrm{o} \mathrm{pH}$ da solução de MNZ para valores de 4,7 e 10. A maior eficiência de degradação foi obtida em $\mathrm{pH} 7$. Isso pode ser explicado devido à variação das cargas elétricas sobre a superficie do MNZ, bem como sobre a superfície do $\mathrm{TiO}_{2}$ em diferentes valores de $\mathrm{pH}$.

Farzadkia e colaboradores (2015), obtiveram resultados semelhantes ao avaliar o efeito do $\mathrm{pH}$. Segundo estes autores, o ponto de carga zero do $\mathrm{TiO}_{2}$ é 6,52 e valor do $\mathrm{pK}_{\mathrm{a}}$ do $\mathrm{MNZ}$ é 2,55. Portanto, em $\mathrm{pH}$ ácido, tanto $\circ \mathrm{TiO}_{2}$ quanto o MNZ estão positivamente carregados, causando efeito negativo para a adsorção do MNZ sobre a superfície do $\mathrm{TiO}_{2}$. $\mathrm{Em}$ pH neutro no entanto, não ocorrem forças de repulsão entre o $\mathrm{TiO}_{2}$ e o MNZ, favorecendo a adsorção do fármaco ao catalisador, aumentando a eficiência na remoção do MNZ da solução. Em pH básico, ambos, $\mathrm{TiO}_{2}$ e MNZ, estão negativamente carregados, dificultando a adsorção do MNZ sobre a superfície do catalisador.

\section{Fotocatálise utilizando $\mathrm{TiO}_{2}$ e luz UV, em presença de $\mathrm{H}_{2} \mathrm{O}_{2}$ e a influência do $\mathrm{pH}$}

O processo fotocatalítico empregando o catalisador $\mathrm{TiO}_{2}$ também foi avaliado na presença de $\mathrm{H}_{2} \mathrm{O}_{2}$. Os resultados obtidos no planejamento experimental $2^{3}$, realizado a fim de avaliar os efeitos das variáveis $\mathrm{pH}$, concentração de $\mathrm{H}_{2} \mathrm{O}_{2}$ e concentração do $\mathrm{TiO}_{2}$ na degradação do MNZ, são apresentados na Tabela 5.
Para analisar a eficiência desse processo de fotodegradação, avaliou-se a \%Red. Os resultados obtidos apresentaram variações de $38,76 \%$ a $71,2 \%$.

A maior taxa de fotodegradação foi de $71,2 \%$, obtida nos maiores níveis das variáveis avaliadas: $\mathrm{pH}$ 10, concentração de $\mathrm{TiO}_{2} 100$ $\mathrm{mg} \cdot \mathrm{L}^{-1}$ e concentração de $\mathrm{H}_{2} \mathrm{O}_{2}$ de $1000 \mathrm{mg} \cdot \mathrm{L}^{-1}$.

Os valores previstos pelo modelo apresentaram boa correlação entre os valores previstos em função dos valores observados para \%Red, conforme apresentados pela Figura 5.

As respostas experimentais baseadas nos valores de \%Red obtidas no planejamento experimental permitiram o desenvolvimento de um modelo estatístico, com ajuste linear, além do cálculo dos efeitos das interações entre os coeficientes associados às variáveis utilizadas no processo de degradação, conforme apresentados na Tabela 6.

Observando os dados expressos na Tabela 6 conclui-se que, ao nível de significância de $5 \%$, os parâmetros $\mathrm{pH}, \mathrm{H}_{2} \mathrm{O}_{2}, \mathrm{TiO}_{2}$, assim como as interações entre os parâmetros $\mathrm{pH} / \mathrm{TiO}_{2}$ e $\mathrm{H}_{2} \mathrm{O}_{2} / \mathrm{TiO}_{2}$ são significativas para o processo, de modo que com um aumento no valor de $\mathrm{pH}$ e na concentração de $\mathrm{H}_{2} \mathrm{O}_{2}$, observa-se uma maior eficiência de degradação, assim como, o pH alcalino e a maior concentração do catalisador $\mathrm{TiO}_{2}$ aumentam a eficiência do processo.

$\mathrm{O}$ valor de $\mathrm{R}^{2}$ de 0,97838 indica que cerca de $97 \%$ da variabilidade na resposta pode ser explicada por meio do modelo calculado.

O ajuste do modelo, considerando apenas os parâmetros significativos do processo, resultaram na Equação 3.

$\%$ Red $=51,598+4,787 \cdot \mathrm{pH}+4,132 .\left[\mathrm{H}_{2} \mathrm{O}_{2}\right]+$ 5,970. $\left[\mathrm{TiO}_{2}\right]+7,300 . \mathrm{pH} \cdot\left[\mathrm{TiO}_{2}\right]-1,920$. $\left[\mathrm{H}_{2} \mathrm{O}_{2}\right] \cdot\left[\mathrm{TiO}_{2}\right]$

O efeito das variáveis significativas e suas interações são verificados aplicando a análise de variância (ANOVA), como apresentado na Tabela 7. Nas condições propostas o $F_{\mathrm{Cal}}(76,426)$ para o modelo é maior que o $F_{\text {Tab }}(6 ; 4 ; 0,05=6,16)$, portanto, o modelo é válido para o nível de significância de $5 \%$. 
Os resultados experimentais obtidos no planejamento e a análise dos parâmetros operacionais do reator de fotodegradação podem ser melhores visualizados no gráfico da superfície de resposta.

$\mathrm{Na}$ Figura 6, foi analisado o efeito das variáveis significativas $\left(\mathrm{pH} \mathrm{e} \mathrm{H}_{2} \mathrm{O}_{2}\right)$ do processo, mantendo-se a terceira variável $\left(\mathrm{TiO}_{2}\right)$ fixa no ponto central estabelecido no planejamento. Observa-se que em pH 10 obtém-se uma melhor eficiência do processo de fotodegradação, assim como, quando utiliza-se a concentração de 1000 $\mathrm{mg} \cdot \mathrm{L}^{-1}$ de $\mathrm{H}_{2} \mathrm{O}_{2}$.

Tabela 10. \%Red após 60 minutos de irradiação, para o MNZ em pH 10

\begin{tabular}{lc} 
Processo & \%Red (\%) \\
\hline $\mathrm{UV} / \mathrm{H}_{2} \mathrm{O}_{2}$ & 52,00 \\
$\mathrm{UV} / \mathrm{TiO}_{2}$ & 62,23 \\
$\mathrm{UV} / \mathrm{TiO}_{2} / \mathrm{H}_{2} \mathrm{O}_{2}$ & 71,20 \\
\hline
\end{tabular}

\section{Avaliação da toxicidade utilizando Artemia salina}

$\mathrm{Na}$ Tabela 11 estão representados os resultados obtidos no ensaio de toxicidade realizados nas condições experimentais que apresentaram melhores taxas de degradação do MNZ, ou seja, $\mathrm{pH} 10,1000 \mathrm{mg} \cdot \mathrm{L}^{-1}$ de $\mathrm{H}_{2} \mathrm{O}_{2}$ e 100 $\mathrm{mg} \cdot \mathrm{L}^{-1}$ de $\mathrm{TiO}_{2}$. Como controle negativo foi utilizado água destilada e como controle positivo uma solução aquosa contendo $\mathrm{MNZ}$ na concentração de $10 \mathrm{mg} \cdot \mathrm{L}^{-1} \mathrm{com} \mathrm{pH}$ ajustado previamente para $10 . \quad \mathrm{O}_{2} \mathrm{O}_{2}$ remanescente após o ensaio de fotocatálise foi eliminado pela adição de uma quantidade catalítica de $\mathrm{MnO}_{2}$.

Tabela 11. Percentagem de mortalidade nos ensaios ecotoxicológicos.

\section{Ensaio ecotoxicológico Mortalidade (\%)}

\begin{tabular}{lc}
\hline Controle negativo & 0 \\
Controle positivo & 90 \\
Tratamento & 20 \\
\hline
\end{tabular}

O percentual de mortalidade obtido no controle positivo foi de $90 \%$, indicando que a solução de MNZ é letal para os organismos testados. Na solução do antibiótico submetida ao processo de fotodegradação, apenas $20 \%$ dos organismos-testes estavam mortos após 24 horas, indicando que o tratamento realizado diminui a toxicidade para $A$. salina.

\section{CONCLUSÕES:}

O processo de degradação do MNZ empregando luz UV em diferentes valores de $\mathrm{pH}$, apresentou maior eficiência e velocidade de reação em $\mathrm{pH}$ 4. Quando se adicionou o $\mathrm{H}_{2} \mathrm{O}_{2}$ ao processo, a maior percentagem de degradação foi em $\mathrm{pH} 10$, utilizando $1000 \mathrm{mg} \cdot \mathrm{L}^{-1}$ de $\mathrm{H}_{2} \mathrm{O}_{2}$, obtendo-se um valor de $k$ equivalente a $1,23 \times 10^{-2}$ $\mathrm{min}^{-1}$,ajustado a uma cinética de pseudo-primeira ordem, muito superior àquele determinado para o processo empregando apenas a luz UV $(k=1,5 \mathrm{x}$ $\left.10^{-3} \min ^{-1}\right)$.

Nos ensaios de fotodegradação utilizando o catalisador $\mathrm{TiO}_{2}$, em presença ou ausência de $\mathrm{H}_{2} \mathrm{O}_{2}$, em diferentes valores de $\mathrm{pH}$, pôde-se verificar, por meio do planejamento experimental $2^{3}$, que os parâmetros $\mathrm{pH}, \mathrm{H}_{2} \mathrm{O}_{2}, \mathrm{TiO}_{2}$, assim como a interação entre os parâmetros $\mathrm{pH}$ e $\mathrm{TiO}_{2}$ e $\mathrm{H}_{2} \mathrm{O}_{2}$ e $\mathrm{TiO}_{2}$ são significativos para o processo, de modo que com um aumento no valor de $\mathrm{pH}$ e na concentração de $\mathrm{H}_{2} \mathrm{O}_{2}$, observa-se uma maior eficiência de degradação, assim como, em pH alcalino e na maior concentração do catalisador $\mathrm{TiO}_{2}$ obtém-se melhor eficiência do processo. As taxas de degradação obtidas quando não se utiliza $\circ \mathrm{H}_{2} \mathrm{O}_{2}$ ao processo de fotocatálise, são maiores em pH 10 e concentração de $\mathrm{TiO}_{2}$ igual a $100 \mathrm{mg} \cdot \mathrm{L}^{-1}$, porém, a eficiência do processo é menor.

No que se refere aos ensaios ecotoxicológicos, houve redução de $80 \%$ na toxicidade após o tratamento realizado em $\mathrm{pH}$ 10, utilizando $1000 \mathrm{mg} \cdot \mathrm{L}^{-1}$ de $\mathrm{H}_{2} \mathrm{O}_{2}$ e o catalisador $\mathrm{TiO}_{2}$ na concentração de100 $\mathrm{mg} \cdot \mathrm{L}^{-1}$.

\section{AGRADECIMENTOS:}

Os autores agradecem a CAPES e a Fundação Araucária o auxílio financeiro concedido.

\section{REFERÊNCIAS:}

1. ANDREOZZI, R.; RAFFAELE, M.; NICKLAS, 
$P$. Pharmaceuticals in STP effluents and their solar photodegradation in aquatic environment. Chemosphere, v. 50, p. 13191330 , http://dx.doi.org/10.1016/S00456535(02)00769-5.

2. BILA, D. M.; DEZOTTI, M.Fármacos no meio ambiente. Química Nova, v. 26, p. 523-530, 2003.

http://dx.doi.org/10.1590/S010040422003000400015.

3. BINIECKA, M.; CAMPANA, P.; IANNILLI, I. The technological and economic management of the environmental variable in the pharmaceutical-chemical industry. Microchemical Journal, v. 79 , p. 325-329, 2005.

http://dx.doi.org/10.1016/j.microc.2004.10.002

4. BOXALL, A. B. A. The environmental side effects of medication. EMBO Reports, v. 12, p. 1110-1116, http://dx.doi.org/10.1038/sj.embor.7400307.

5. ÇAVAS, T.; ERGENE-GÖZÜKARA, S. Induction of micronuclei and nuclear abnormalities in Oreochromis niloticus following exposure to petroleum refinery and chromium processing plants effluents. Aquatic Toxicology, v. 74, p. 264-271, 2005.

http://dx.doi.org/10.1016/j.aquatox.2005.06.00 1.

6. CETESB. Água do Mar: Teste de toxicidade aguda com Artemia salina. Norma CETESB L05.021/1987.

7. CISNEROS, R. L.; ESPINOZA, A. G.; LITTER, M. I.Photodegradation of an azo dye of the textile industry. Chemosphere, v. 48, p. 393399, 2002.

http://dx.doi.org/10.1016/S00456535(02)00117-0.

8. DANTAS, R. F.; ROSSITER, O.; TEIXEIRA, R. K. A.; SIMOES, A. S. M.; SILVA, V. L. Direct UV photolysis of propranolol and metronidazole in aqueous solution. Chemical Engineering Journal, v. 158, p. 143-147, 2010. http://dx.doi.org/10.1016/j.cej.2009.12.017.

9. DÍAZ-CRUZ, M. S.; DE ALDA, M. J. L.; BARCELÓ, D. Environmental behavior and analysis of veterinary and human drugs in soils, sediments and sludge. TrAC Trends Analytical Chemistry, v. 22, p. 340-351, 2003. http://dx.doi.org/10.1016/S01659936(03)00603-4.

10. FARZADKIA, M.; ESRAFILI, A.; MOHAMMAD ALI, B.; SHAHAMAT, Y. D.; OKHOVAT, N. Degradation of metronidazole in aqueous solution by nano- $\mathrm{ZnO} / \mathrm{UV}$ photocatalytic process. Desalination and Water Treatment, v. 52, p. 4947-4952, 2014. http://dx.doi.org/10.1080/19443994.2013.8103 22.

11. FARZADKIA, M.; BAZRAFSHAN, E.; ESRAFILI, A.; YANG, J.; SHIRZAD-SIBONI, M. Photocatalytic degradation of Metronidazole with illuminated $\mathrm{TiO}_{2}$ nanoparticle. Journal of Environmental Health Science and Engineering, v. 13, p. 35-43, 2015. http://dx.doi.org/10.1186/s40201-0150194-y.

12. FUJISHIMA, A.; ZHANG, X.; TRYK, D. A. $\mathrm{TiO}_{2}$ photocatalysis and related surface phenomena. Surface Science Reports, v. 63, p. 515-582, 2008.

http://dx.doi.org/10.1016/j.surfrep.2008.10.001

13. HALLING-SØRENSEN, B.; NORS NIELSEN, S.; LANZKY, P. F.; INGERSLEV, F.; HOLTEN LÜTZHØFT, H. C.; JØRGENSEN, S. E. Occurrence, fate and effects of pharmaceutical substances in the environment - A review. Chemosphere, v. 36, p. 357-393, 1998.

http://dx.doi.org/10.1016/S0045-

6535(97)00354-8.

14. KASPRZYK-HORDERN, B.; DINSDALE, R. M.; GUWY, A. J. The occurrence of pharmaceuticals, personal care products, endocrine disruptors and illicit drugs in surface water in South Wales, UK. Water Research, v. 42, p. 3498-3518, 2008.

http://dx.doi.org/10.1016/j.watres.2008.04.026.

15. KÜMMERER, K. The presence of pharmaceuticals in the environment due to human use - present knowledge and future challenges. Journal of Environmental Management, v. 90, p. 2354-2366, 2009. http://dx.doi.org/10.1016/j.jenvman.2009.01.02 3.

16. LINDBERG, R.; JARNHEIMER, P. A.; OLSEN, B.; JOHANSSON, M.; TYSKLIND, M. Determination of antibiotic substances in hospital sewage water using solid phase extraction and liquid chromatography/mass spectrometry and group analogue internal standards. Chemosphere, v. 57, p. 1479-1488, 2004.

http://dx.doi.org/10.1016/j.chemosphere.2004. 09.015.

17. MELO, S. A. S.; TROVÓ, A. G.; BAUTITZ, I. R.; NOGUEIRA, R. F. P. Degradação de fármacos residuais por processos oxidativos avançados. Química Nova, v. 32, p. 188-197, 2009. http://dx.doi.org/10.1590/S0100- 
40422009000100034.

18. MEYER, B. N.; FERRIGNI, N. R.; PUTNAM, J. E.; JACOBSEN, L. B.; NICHOLS, D. E.; McLAUGHLIN, J. L. Brine shrimp: a convenient general bioassay for active plant constituents. Journal of Medical Plant Research, v.45, p. 31-34, 1982.

http://dx.doi.org/10.1055/s-2007-971236.

19. NASCIMENTO, I. A.; ARAÚJO, M. M. S. Testes Ecotoxicológicos Marinhos: Análise de Sensibilidade. Ecotoxicology and Environmental. Restoration, v. 2, p. 4147,1999.

20. NOGUEIRA, R. F. P.; ALBERICI, R. M.; JARDIM, W. F. Heterogeneous photocatalysis: an emerging technology for remediation of VOC contaminated environments. Ciência e Cultura, v. 49, p. 14-24, 1997.

21. OKHOVAT, N.; HASHEMI, M.; GOLPAYEGANI, A. A. J. Photocatalytic decomposition of Metronidazole in aqueous solutions using titanium dioxide nanoparticles. Journal of Materials and Environmental Science, v. 6, p. 792-799, 2015.

22. PRADOS-JOYA, G.; SÁNCHEZ-POLO, M.; RIVERA-UTRILLA, J.; FERRO-GARCÍA, $M$. Photodegradation of the antibiotics nitroimidazoles in aqueous solution by ultraviolet radiation. Water Research, v. 45, p. 393-403, 2011. http://dx.doi.org/10.1016/j.watres.2010.08.015.

23. REIS FILHO, R. W.; ARAÚJO, J. C.; VIEIRA, E. M. Hormônios sexuais estrógenos: contaminantes bioativos. Química Nova, v. 29, p. 817, 2006. http://dx.doi.org/10.1590/S010040422006000400032.

24. SÁNCHEZ-POLO, M.; RIVERA-UTRILLA, J.; PRADOS-JOYA, G.; OCAMPO-PÉREZ, R. $J$. Metronidazole photodegradation in aqueous solution by using photosensitizers and hydrogen peroxide. Journal of Chemical Technology and Biotechnology, v. 87, p. 12021208 , 2012. http://dx.doi.org/10.1002/jctb.3750.

25. SCHNEIDER, M. V.; ROSA, M. F.; LOBO, V. S.; BARICCATTI, R. A. Photocatalytical degradation of bentazone with $\mathrm{TiO}_{2}$. Engenharia Sanitária Ambiental, v. 19, p. 6166, 2014.

26. SHEMER, H.; KUNUKCU, Y. K.; LINDEN, K. G.Degradation of the pharmaceutical metronidazole via UV, Fenton and photoFenton processes. Chemosphere, v. 63, p. 269-276, 2006.

http://dx.doi.org/10.1016/j.chemosphere.2005.
07.029 .

27. SILVA, S. S.; MAGALHÃES, F.; SANSIVIERO, M. T. C. NANOCOMPÓSITOS SEMICONDUTORES $\mathrm{ZnO} / \mathrm{TiO}_{2}$. TESTES FOTOCATALÍTICOS. Química Nova, v. 33, p. 85-89, 2010.

http://dx.doi.org/10.1590/S0100-

40422010000100016

28. SORENSEN, B.H., NIELSEN, S.N., LANZKY, P.F., INGERSLEV, F., LUTZHOFT, H.C.H., JORGENSEN, S.E. Occurence, fate and effects of pharmaceutical substances in the environment- $A$ review. Chemosphere, $v$. 36, p.357-393, 1998.

http://dx.doi.org/10.1016/S0045-

6535(97)00354-8.

29. VEIGA, L. F.; VITAL, N. 2002, Teste de toxicidade aguda com $\mathrm{O}$ microcrustáceo Artemia sp. In: Nascimento, I. A.; Sousa, E. C. P.; Nipper, M. Métodos em ecotoxicologia marinha. Aplicações no Brasil. p.111-122.

30. VEIGA, L.F.; VITAL, N.A.; PORTELA, M. R.; OLIVEIRA, F.F. (1989). Avaliação de faixa de sensibilidade de Artemia salina ao Lauril Sulfato de Sódio. PETROBRÁS/CENPES/SUPESQ/DITER, 64p. il. (Projeto 04.05.27). Rio de Janeiro.

31. VOGEL, A. I. Análise Química Quantitativa. 5th ed.; JC Editora: Rio de Janeiro, Brasil, 1992.

32. XAVIER, L. F. W.; MOREIRA, I. M. N.S.; HIGARASHI, M. M.; MOREIRA, J.C.; FERREIRA, L. F. V.; OLIVEIRA, A. S. Fotodegradação de Hidrocarbonetos Policíclicos Aromáticos em Placas de Sílica Impregnadas com Dióxido de Titânio. Química Nova v. 28, p. 409-413, 2005. http://dx.doi.org/10.1590/S010040422005000300009 


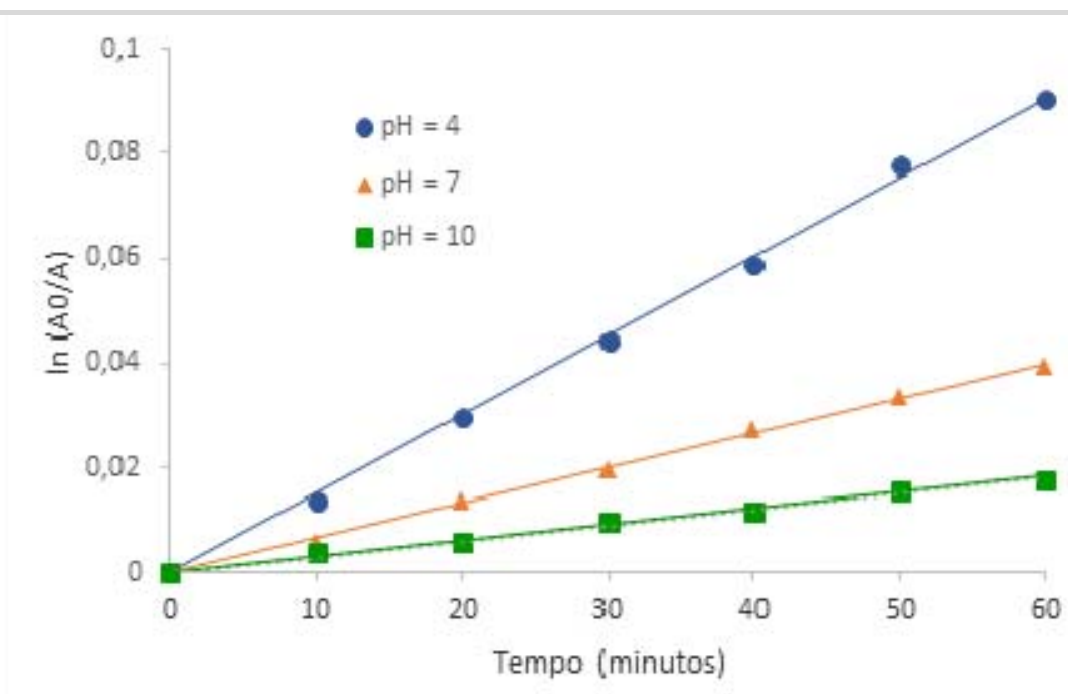

Figura 2. Ajustes lineares dos pontos do gráfico de $\ln \left(A_{0} / A\right)$ vs tempo $(\mathrm{min})$ para as amostras com diferentes valores de $\mathrm{pH}$

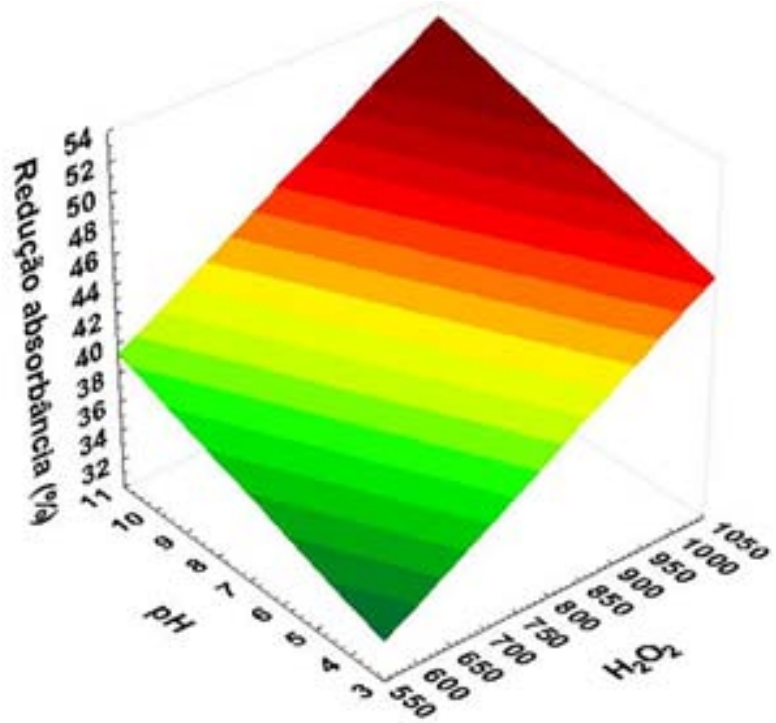

Figura 3. Superfície de resposta de percentagem de redução da absorbância para as variáveis $\mathrm{pH}$ e concentração de $\mathrm{H}_{2} \mathrm{O}_{2}$ 


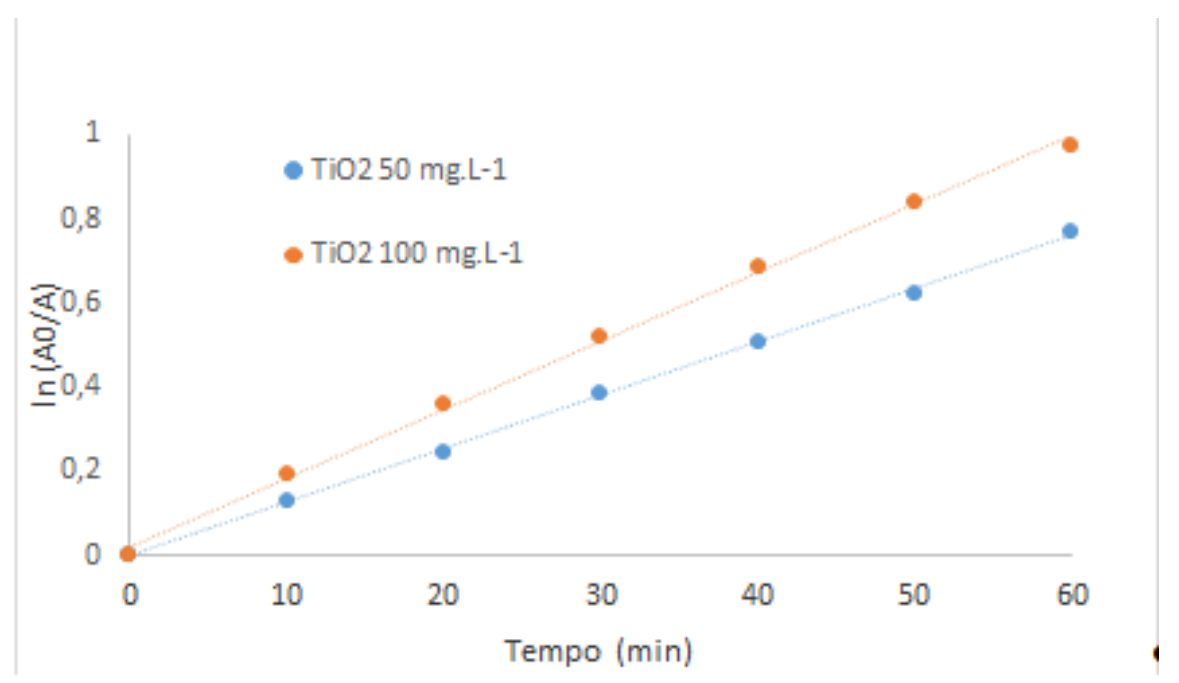

Figura 4. Ajustes lineares de $\ln \left(\mathrm{A}_{0} / \mathrm{A}\right)$ vs tempo para duas concentrações de $\mathrm{TiO}_{2}$ em pH 10.

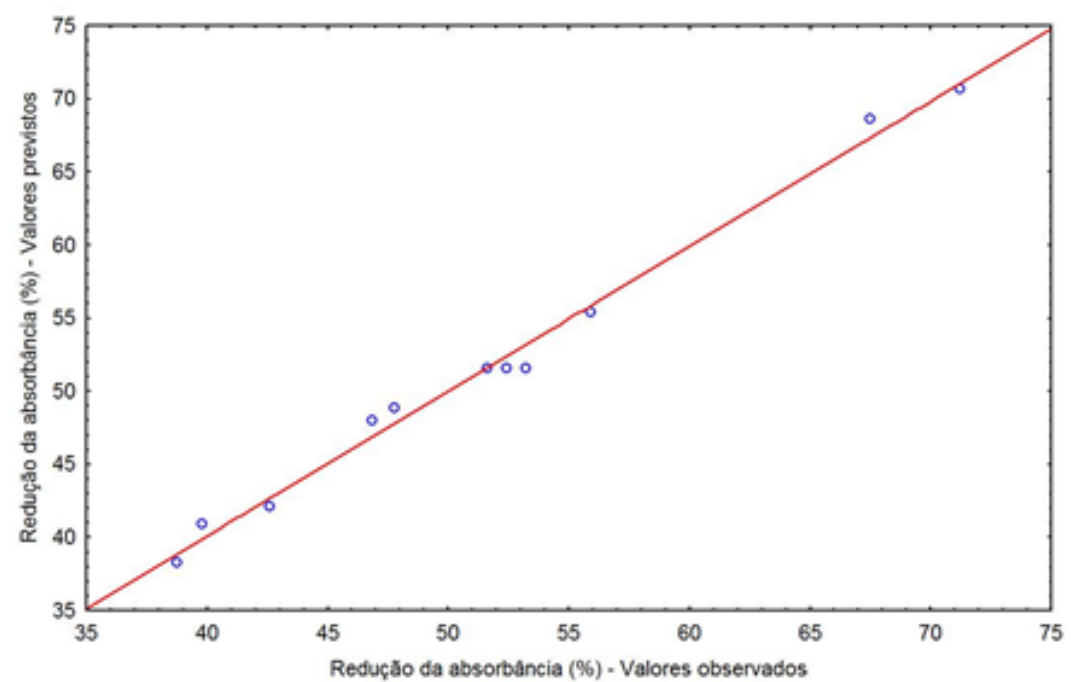

Figura 5. Valores previstos para o processo de fotodegradação em função dos valores observados para a redução da absorbância

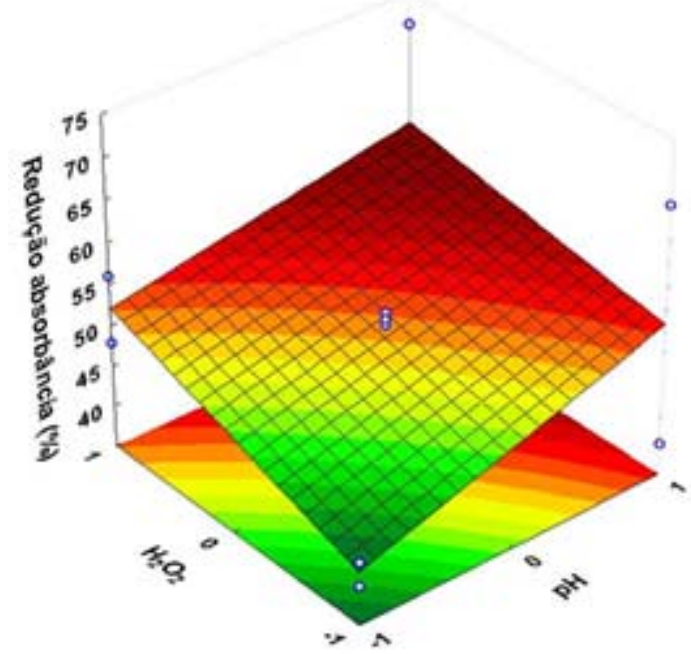

Figura 6. Superfície de resposta mantendo-se fixa no ponto central $\left(75 \mathrm{mg} \cdot \mathrm{L}^{-1}\right)$ a variável $\mathrm{TiO}_{2}$

PERIÓDICO TCHÊ QUÍMICA • www.periodico.tchequimica.com • Vol. 14 N. 28.

•ISSN 1806-0374 (impresso) • ISSN 1806-9827 (CD-ROM) • ISSN 2179-0302 (meio eletrônico) 
Tabela 1. Níveis das variáveis $\left(\mathrm{TiO}_{2}, \mathrm{H}_{2} \mathrm{O}_{2} \mathrm{e} \mathrm{pH}\right)$ utilizadas no planejamento experimental.

\begin{tabular}{lccc}
\hline Variável & \multicolumn{3}{c}{ Níveis } \\
\hline $\mathrm{TiO}_{2}\left(\mathrm{mg} \cdot \mathrm{L}^{-1}\right)$ & 50 & 75 & 100 \\
$\mathrm{H}_{2} \mathrm{O}_{2}\left(\mathrm{mg} \cdot \mathrm{L}^{-1}\right)$ & 600 & 800 & 1000 \\
$\mathrm{pH}$ & 4,0 & 7,0 & 10,0 \\
\hline
\end{tabular}

Tabela 2.Valores de percentual de redução da banda, constantes cinéticas e $R^{2}$ para os experimentos de fotólise utilizando luz UV e a influência do $\mathrm{pH}$, após 60 minutos de irradiação.

\begin{tabular}{cccc}
\hline $\mathrm{pH}$ & $\% \operatorname{Red}(\%)$ & $\mathrm{k}\left(\mathrm{min}^{-1}\right)$ & $\mathrm{R}^{2}$ \\
\hline 4,0 & 8,66 & $1,5 \times 10^{-3}$ & 0,998 \\
7,0 & 3,87 & $6,69 \times 10^{-4}$ & 0,998 \\
10,0 & 1,77 & $2,97 \times 10^{-4}$ & 0,992 \\
\hline
\end{tabular}

Tabela 3. Valores de percentual de redução da absorbância, constantes cinéticas e $\mathrm{R}^{2}$ para os experimentos de fotólise utilizando $\mathrm{H}_{2} \mathrm{O}_{2}$ e luz UV e a influência do pH, após 60 minutos de irradiação

\begin{tabular}{|c|c|c|c|c|}
\hline $\mathrm{pH}$ & {$\left[\mathrm{H}_{2} \mathrm{O}_{2}\right]\left(\mathrm{mg} \cdot \mathrm{L}^{-1}\right)$} & $\% \operatorname{Red}(\%)$ & $k\left(\min ^{-1}\right)$ & $\mathrm{R}^{2}$ \\
\hline \multirow{3}{*}{4,0} & 0 & 8,66 & $1,5 \times 10^{-3}$ & 0,998 \\
\hline & 600 & 36 & $7,43 \times 10^{-3}$ & 0,998 \\
\hline & 1000 & 45 & $9,79 \times 10^{-3}$ & 0,998 \\
\hline \multirow{3}{*}{7,0} & 0 & 3,87 & $6,69 \times 10^{-4}$ & 0,998 \\
\hline & 600 & 37 & $7,63 \times 10^{-3}$ & 0,998 \\
\hline & 1000 & 48 & $1,10 \times 10^{-2}$ & 0,998 \\
\hline \multirow{3}{*}{10,0} & 0 & 1,67 & $2,97 \times 10^{-4}$ & 0,992 \\
\hline & 600 & 39 & $8,37 \times 10^{-3}$ & 0,990 \\
\hline & 1000 & 52 & $1,23 \times 10^{-2}$ & 0,990 \\
\hline
\end{tabular}


Tabela 4. Valores de porcentagem de redução da absorbância (\%Red) após 60 minutos de irradiação, $k$ e $\mathrm{R}^{2}$, para o catalisador $\mathrm{TiO}_{2}$.

\begin{tabular}{|c|c|c|c|}
\hline$\left[\mathrm{TiO}_{2}\right]\left(\mathrm{mg} \cdot \mathrm{L}^{-1}\right)$ & \multicolumn{2}{|c|}{$\% \operatorname{Red}(\%)\left(\min ^{-1}\right)$} & $\mathrm{R}^{2}$ \\
\hline 50 & 53,63 & $1,27 \times 10^{-2}$ & 0,999 \\
\hline 100 & 62,23 & $1,62 \times 10^{-2}$ & 0,998 \\
\hline
\end{tabular}

Tabela 5. Matriz de planejamento fatorial $2^{3}$ com resultado de percentagem de redução da absorbância em função do $\mathrm{pH}$ da solução, concentração de peróxido de hidrogênio e concentração de dióxido de titânio.

\begin{tabular}{ccccc} 
Experimento & $\mathrm{pH}$ & $\mathrm{H}_{2} \mathrm{O}_{2}\left(\mathrm{mg} \cdot \mathrm{L}^{-1}\right)$ & $\mathrm{TiO}_{2}\left(\mathrm{mg} \cdot \mathrm{L}^{-1}\right)$ & $\% \operatorname{Red}(\%)$ \\
\hline P1 & -1 & -1 & -1 & 39,78 \\
P2 & -1 & -1 & 1 & 42,60 \\
P3 & -1 & 1 & -1 & 55,89 \\
P4 & -1 & 1 & 1 & 47,75 \\
P5 & 1 & -1 & -1 & 38,76 \\
P6 & 1 & -1 & 1 & 67,50 \\
P7 & 1 & 1 & -1 & 46,86 \\
P8 & 1 & 1 & 1 & 71,20 \\
P9 & 0 & 0 & 0 & 51,63 \\
P10 & 0 & 0 & 0 & 53,20 \\
P11 & 0 & 0 & 0 & 52,41 \\
\hline
\end{tabular}

Tabela 6. Efeitos e coeficientes das variáveis principais e das interações do processo de fotodegradação com irradiação artificial para redução da absorbância.

\begin{tabular}{llllll}
\hline $\begin{array}{l}\text { Variáveis e } \\
\text { interações }\end{array}$ & $\begin{array}{l}\text { Efeito da } \\
\text { Variável }\end{array}$ & $\begin{array}{l}\text { Coeficiente do } \\
\text { modelo }\end{array}$ & $\begin{array}{l}\text { Erro padrão do } \\
\text { coeficiente }\end{array}$ & $t_{\text {exp }}(4)$ & $p$-valor \\
\hline Intercepto & $\mathbf{5 1 , 5 9 8}$ & $\mathbf{5 1 , 5 9 8}$ & $\mathbf{0 , 4 6 1}$ & $\mathbf{1 1 1 , 9 1 6}$ & $\mathbf{0 , 0 0 0}$ \\
$\mathrm{pH}$ & $\mathbf{9 , 5 7 5}$ & $\mathbf{4 , 7 8 7}$ & $\mathbf{0 , 5 4 0}$ & $\mathbf{8 , 8 5 5}$ & $\mathbf{0 , 0 0 0}$ \\
$\mathrm{H}_{2} \mathrm{O}_{2}$ & $\mathbf{8 , 2 6 5}$ & $\mathbf{4 , 1 3 2}$ & $\mathbf{0 , 5 4 0}$ & $\mathbf{7 , 6 4 4}$ & $\mathbf{0 , 0 0 1}$ \\
$\mathrm{TiO}_{2}$ & $\mathbf{1 1 , 9 4 0}$ & $\mathbf{5 , 9 7 0}$ & $\mathbf{0 , 5 4 0}$ & $\mathbf{1 1 , 0 4 2}$ & $\mathbf{0 , 0 0 0}$ \\
$\mathrm{pH} / \mathrm{H}_{2} \mathrm{O}_{2}$ & $-2,365$ & $-1,182$ & 0,540 & $-2,187$ & 0,093 \\
$\mathrm{pH} / \mathrm{TiO}_{2}$ & $\mathbf{1 4 , 6 0 0}$ & $\mathbf{7 , 3 0 0}$ & $\mathbf{0 , 5 4 0}$ & $\mathbf{1 3 , 5 0 3}$ & $\mathbf{0 , 0 0 0}$ \\
$\mathrm{H}_{2} \mathrm{O}_{2} / \mathrm{TiO}_{2}$ & $\mathbf{- 3 , 8 4 0}$ & $-1,920$ & $\mathbf{0 , 5 4 0}$ & $-3,551$ & $\mathbf{0 , 0 2 3}$ \\
\hline
\end{tabular}

$R^{2}=0,97838$ 
Tabela 7. Análise de variância do modelo previsto para o processo de fotodegradação na redução da absorbância.

\begin{tabular}{cccccc}
\hline $\begin{array}{c}\text { Fonte de } \\
\text { Variação }\end{array}$ & $\begin{array}{c}\text { Soma } \\
\text { Quadrática }\end{array}$ & $\begin{array}{c}\mathrm{N}^{\circ} \text { de graus } \\
\text { de liberdade }\end{array}$ & $\begin{array}{c}\text { Média } \\
\text { Quadrática }\end{array}$ & $\mathrm{F}$ & $p$-valor \\
\hline $\mathrm{pH}$ & $\mathbf{1 8 3 , 3 6 1}$ & $\mathbf{1}$ & $\mathbf{1 8 3 , 3 6 1}$ & $\mathbf{7 8 , 4 2 1}$ & $\mathbf{0 , 0 0 0}$ \\
$\mathrm{H}_{2} \mathrm{O}_{2}$ & $\mathbf{1 3 6 , 6 2 0}$ & $\mathbf{1}$ & $\mathbf{1 3 6 , 6 2 0}$ & $\mathbf{5 8 , 4 3 0}$ & $\mathbf{0 , 0 0 1}$ \\
& $\mathbf{2 8 5 , 1 2 7}$ & $\mathbf{1}$ & $\mathbf{2 8 5 , 1 2 7}$ & $\mathbf{1 2 1 , 9 4 5}$ & $\mathbf{0 , 0 0 0}$ \\
$\mathrm{TiO}_{2}$ & 11,186 & 1 & 11,186 & 4,784 & 0,093 \\
$\mathrm{pH} / \mathrm{H}_{2} \mathrm{O}_{2}$ & $\mathbf{4 2 6 , 3 2 0}$ & $\mathbf{1}$ & $\mathbf{4 2 6 , 3 2 0}$ & $\mathbf{1 8 2 , 3 3 1}$ & $\mathbf{0 , 0 0 0}$ \\
$\mathrm{pH} / \mathrm{TiO}_{2}$ & $\mathbf{2 9 , 4 9 1}$ & $\mathbf{1}$ & $\mathbf{2 9 , 4 9 1}$ & $\mathbf{1 2 , 6 1 3}$ & $\mathbf{0 , 0 2 3}$ \\
$\mathrm{H}_{2} \mathrm{O}_{2} / \mathrm{TiO}_{2}$ & 9,353 & 4 & 2,338 & & \\
Erro Padrão & 1081,459 & 10 & &
\end{tabular}

PERIÓDICO TCHÊ QUÍMICA • www.periodico.tchequimica.com • Vol. 14 N. 28.

- ISSN 1806-0374 (impresso) • ISSN 1806-9827 (CD-ROM) • ISSN 2179-0302 (meio eletrônico)

(C) 2017. Porto Alegre, RS. Brasil 